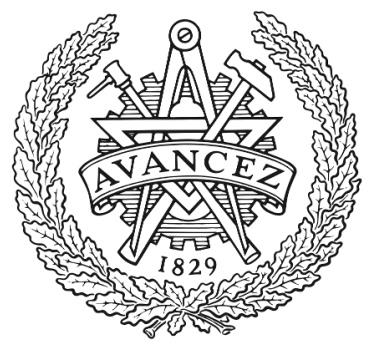

CHALMERS

UNIVERSITY OF TECHNOLOGY

\title{
Design of Variable-Speed Dish-Stirling Solar-Thermal Power Plant for Maximum Energy Harness
}

Downloaded from: https://research.chalmers.se, 2023-04-26 09:04 UTC

Citation for the original published paper (version of record):

Li, Y., Choi, S., Yang, C. et al (2015). Design of Variable-Speed Dish-Stirling Solar-Thermal Power Plant for Maximum Energy Harness. IEEE Transactions on Energy Conversion, 30(1): 394-403. http://dx.doi.org/10.1109/TEC.2014.2354346

N.B. When citing this work, cite the original published paper.

(O2015 IEEE. Personal use of this material is permitted.

However, permission to reprint/republish this material for advertising or promotional purposes 


\title{
Design of Variable-Speed Dish-Stirling Solar-Thermal Power Plant for Maximum Energy Harness
}

\author{
Yang Li, Student Member, IEEE, San Shing Choi, Senior Member, IEEE, \\ Chun Yang, and Feng Wei, Student Member, IEEE
}

\begin{abstract}
Analysis on a developed dynamic model of the dishStirling (DS) system shows that maximum solar energy harness can be realized through controlling the Stirling engine speed. Towards this end, a control scheme is proposed for the doublyfed induction generator coupled to the DS system, as a means to achieve maximum power point tracking as the solar insolation level varies. Furthermore, the adopted fuzzy supervisory control technique is shown to be effective in controlling the temperature of the receiver in the DS system as the speed changes. Simulation results and experimental measurements validate the maximum energy harness ability of the proposed variable-speed DS solarthermal system.
\end{abstract}

Index Terms-Dish-Stirling system, solar-thermal power generation, temperature control.

\section{INTRODUCTION}

D ISH-STIRLING (DS) solar-thermal power generation system involves the use of parabolic dish-like reflector to concentrate sunlight to a small area located at the focal point of the mirrors [1]. A hollow chamber, called the receiver and placed at the focal point, becomes the heat source for a Stirling engine. Stirling engine is a type of closed-cycle external heat engine and is capable of operating at high efficiency and it releases no emissions. Unfortunately, the often random and uncontrollable nature of solar irradiance makes the control of the harnessed energy most challenging. Appropriate system design and operations of the DS power plant are called for. In this connection, the development of suitable DS model for the power plant and control system has been reported in [2]-[8]. In the authors' previous work [2], the steady-state relationship between the engine speed and the generated power has been derived. It shows that harnessed energy from the sun can be maximized if variable-speed operation of the DS system can be realized. As shall be shown in latter sections, variations of the engine speed would impact negatively on the control of the temperature of the receiver: strict control of the temperature is crucial to ensure safe and effective operations of the DS system [2]-[4]. Review of the open literature indicates that there are very few reported works on the variable-speed operation of grid-connected DS system as no suitable mathematical model

Yang Li, San Shing Choi, and Feng Wei are with the School of Electrical and Electronic Engineering, Nanyang Technological University, Singapore 639798 (e-mail: yangli@ieee.org; esschoi@ntu.edu.sg; weif0004@ntu.edu.sg).

Chun Yang is with the School of Mechanical and Aerospace Engineering, Nanyang Technological University, Singapore 639798 (e-mail: mcyang@ntu.edu.sg). has been derived. Existing models of the prime mover proved to be either too complex [5]-[7] or so simplified that they are unsuitable for use in power system studies [8]. Furthermore, in adopting variable-speed operations for the DS system, doubly-fed induction generator (DFIG) is attractive due to its economic advantage over permanent magnet synchronous generator (PMSG). While reported works on speed and power control of DFIG for wind turbines is abound, see e.g. [9], the present investigation shall show that the design of control scheme for such a DS-DFIG system is more complex because the power regulating ability of the generating system will be impacted by the receiver operating temperature. The approach described in [8] ignores the temperature effects and as a result, it can lead to unrealistic prediction of the steady-state and dynamic performances of the DS system.

Accordingly, Section II introduces the overall system configuration of the proposed DS-DFIG power plant. By adopting a more complete analytical representation of the Stirling engine, an improved model of the DS-DFIG system is derived. Based on the steady-state analysis of the developed model, speed and current control of the DFIG is considered in Section III. A fuzzy supervisory scheme for the DS-DFIG is then selected from the possible temperature control techniques considered in Section IV. Numerical examples and experimental results are included in Sections V and VI respectively to validate the maximum energy harnessing ability of the proposed scheme.

\section{Development of the DS-DFIG System Model}

In the DS solar-thermal power generation scheme considered in [2], [4], [6], the dish and Stirling engine function as heat engine to convert solar energy into mechanical energy to drive a constant-speed induction generator. In contrast, in the variable-speed DS system equipped with DFIG considered in the present investigation, a back-to-back converter is added to connect the rotor windings of the DFIG with the grid through a rotor-side converter (RSC), a grid-side converter (GSC), a smoothing dc-link and a LC filter, as shown in Fig. 1. Although the configuration of the generator is similar to that commonly seen in wind power generation system, the challenge is on developing a credible model for the prime mover of the generator, i.e., the dish-Stirling engine. In the following section, a model for the prime mover is derived from basic heat transfer and thermodynamics principles. This 


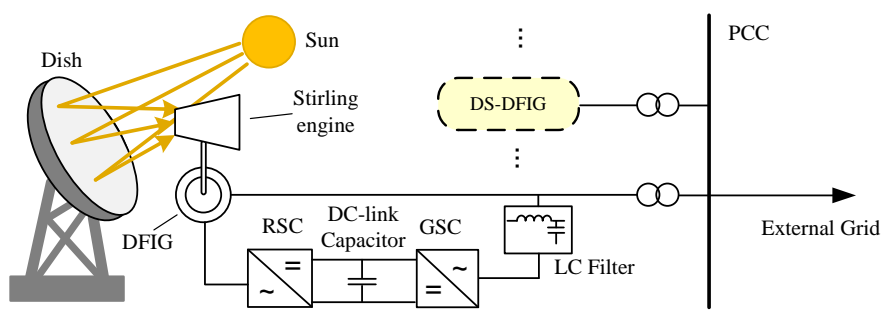

Fig. 1. Schematic diagram of one DS-DFIG unit in a large-scale solar-thermal power plant.

is unlike [8] where the authors have utilized a simple first-order transfer function to model the DS system, and without clear justification on how the model can be sufficiently accurate for the purpose of designing the control system for the DS system.

\section{A. An Improved Dish-Stirling Engine Model}

In the previous work [2], a normalized average-value model of the double-acting kinematic Stirling engine was derived under constant-speed operation. The model is intended for use in conjunction with squirrel-cage induction generator. The present investigation is to include variable-speed operation of the Stirling engine and hence, the model needs to be modified. Also, the assumption made in [2] pertaining to the variations of the temperature of the receiver of the Stirling engine being much slower than that of the pressure change is now removed. Starting from the mass flow equations of the ideal adiabatic model of the Stirling engine, the rather involved derivation in Appendix A shows the following equations can be obtained:

$$
\begin{gathered}
\dot{Q}_{h}=\eta_{h} \dot{Q}_{h, \text { adi }}=\eta_{h}\left[K_{h} p_{\text {mean }} \omega_{m}+A(g A)+C p_{\text {mean }} \dot{T}_{h}\right] \\
P_{m}=\tau_{m} \omega_{m}=\eta_{m} P_{m, \text { adi }}=\eta_{m} K_{m} p_{\text {mean }} \omega_{m} \\
g A=\dot{M}=\dot{p}_{\text {mean }}
\end{gathered}
$$

where $\dot{Q}_{h}$ and $P_{m}$ are the absorbed heat flow rate and generated mechanical power of the Stirling engine respectively, $p_{\text {mean }}$ is the mean pressure of the working gas, $\omega_{m}$ is the engine speed, $g A$ is the net gas flow rate from the high- and lowpressure tanks to the engine cylinders, $M$ is the total mass of the working gas in the cylinders and $T_{h}$ is the temperature of the receiver/absorber. $K_{h}, K_{m}, A$, and $C$ are constants, as defined in the appendix. Their parametric values depend on the physical dimensions of the Stirling engine and are expressed in terms of the selected base values also shown in the appendix.

As explained in [2], various types of thermal and mechanical losses have not been considered in the ideal adiabatic analysis. Hence, thermal efficiency coefficients $\eta_{h}$ and $\eta_{m}$, which are functions of $p_{\text {mean }}$ and $\omega_{m}$, have been incorporated in (1) and (2) to yield a more realistic steady-state characterization of the Stirling engine. The efficiency coefficients are expressed as

$$
\begin{aligned}
& \eta_{h}=\frac{\dot{Q}_{h}(0)}{\dot{Q}_{h, \text { adi }}(0)}=\frac{\sum_{i=0}^{1} \sum_{j=0}^{1} a_{i j} p_{\text {mean }}^{i} \omega_{m}^{j}}{K_{h} p_{\text {mean }} \omega_{m}} \\
& \eta_{m}=\frac{P_{m}(0)}{P_{m, \text { adi }}(0)}=\frac{\sum_{i=0}^{1} \sum_{j=0}^{2} b_{i j} p_{\text {mean }}^{i} \omega_{m}^{j}}{K_{m} p_{\text {mean }} \omega_{m}}
\end{aligned}
$$

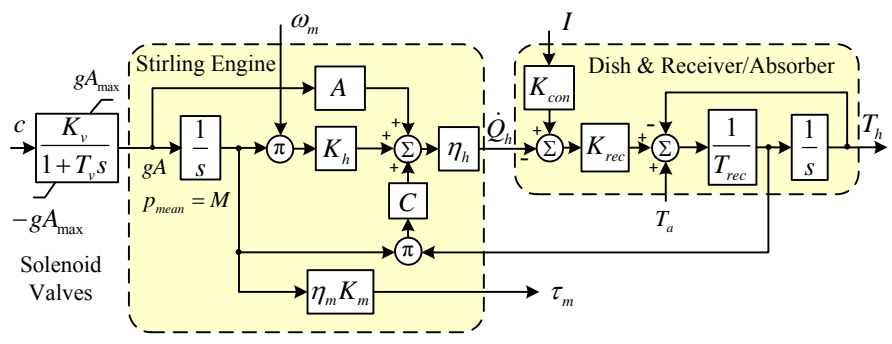

Fig. 2. Block diagram of the normalized average-value model of the DS system.

where $a_{i j}$ and $b_{i j}$ are the multivariate polynomial (MP) coefficients pertaining to the actual input and output powers of the Stirling engine respectively.

The receiver/absorber temperature $T_{h}$ is affected by the input solar insolation $I$ and absorbed heat $\dot{Q}_{h}$ of the Stirling engine. A part of the thermal energy shall be lost to the atmosphere through heat transfer. The equation governing this dynamic is

$$
T_{\text {rec }} \dot{T}_{h}=K_{\text {rec }}\left(K_{\text {con }} I-\dot{Q}_{h}\right)-\left(T_{h}-T_{a}\right)
$$

where $T_{a}$ is the normalized temperature of the atmosphere [2]. The constants $T_{\text {rec }}, K_{\text {rec }}$, and $K_{\text {con }}$ are governed by the efficiency of the dish, projection area, thermal characteristics of the absorber, among other factors. Through mean pressure control technique, $\dot{Q}_{h}$ is regulated by varying the pressure of the working gas via supplying or dumping the gas using fastresponding solenoid valves. The process is modeled as

$$
T_{v}(g \dot{A})=-(g A)+K_{v} c
$$

where $K_{v}$ and $T_{v}$ are the gain and time constants of the solenoid valves, and $c$ is the input command of the solenoid valves to control the working gas. Based on (1)-(7), a more accurate average-value model of the dish and Stirling engine compared to that shown in [2] is derived and is as shown in Fig. 2.

\section{B. Modeling of DFIG \& RSC}

As the purpose of the present investigation is to design temperature and speed control systems for the DS-DFIG, the model used in [8] for the induction machine and converter can be simplified by ignoring the much faster electromagnetic dynamics. Traditionally, current control forms the inner loop of the double-loop feedback PI control of DFIG whereas the outer loop is for speed/power control. With appropriate decoupling techniques, the design of the inner and outer loops PI controllers can be carried out separately because the dynamics of the inner (electromagnetic) loop are much faster than that of the outer (electromechanical and thermo-mechanical) loop. Thus, in the design of the speed and temperature controllers, the inner loop can be modeled as an ideal current or torque source. The electromagnetic torque $\tau_{e}$ is assumed to be the same as its reference value, i.e., $\tau_{e}^{*}=\tau_{e}$. As a result, the electromechanical dynamics of the DFIG contain only the equation of motion

$$
2 H^{\prime} \dot{\omega}_{m}=\tau_{m}-\tau_{e}-F^{\prime} \omega_{m}
$$


where $H^{\prime}$ and $F^{\prime}$ are the equivalent inertia constant and friction constant of a single-lumped-mass shaft system respectively. $H^{\prime}, F^{\prime}$ and the torque quantities have been converted to the same bases as those are used in the normalized model of the DS developed in Appendix A.

\section{Linearized Model}

The DS-DFIG system is expected to operate under constant changes in the solar irradiance $I$. Thus it would be meaningful to firstly construct a suitable model which describes the behavior of the solar-thermal plant under such smalldisturbance condition. For a small displacement about any arbitrary operating point $I=I_{0}, p_{\text {mean }}=p_{0}, \omega_{m}=\omega_{m 0}$, and $T_{h}=T_{h 0}$, using (1), (4), and (6), one can obtain the small-signal equation governing the working gas pressure and heat transfer dynamics

$$
\begin{aligned}
& T_{\text {rec }}^{\prime} \Delta \dot{T}_{h}+\Delta T_{h}=K_{\text {rec }}\left[K_{\text {con }} \Delta I-\eta_{h 0} A \Delta p_{\text {mean }}\right. \\
& \left.-\left(a_{10}+a_{11} \omega_{m 0}\right) \Delta p_{\text {mean }}-\left(a_{01}+a_{11} p_{0}\right) \Delta \omega_{m}\right]
\end{aligned}
$$

where $\eta_{h 0}$ is the corresponding thermal efficiency at the operating point. From (9), the following transfer functions can be obtained:

$$
\begin{gathered}
\frac{\Delta T_{h}(s)}{\Delta p_{\text {mean }}(s)}=\frac{-K_{\mathrm{rec}} K_{\mathrm{hp}}\left(1-T_{\mathrm{sel} 1}\right)}{1+T_{\mathrm{rec}}^{\prime} s} \\
\frac{\Delta T_{h}(s)}{\Delta \omega_{m}(s)}=\frac{-K_{\mathrm{rec}} K_{\mathrm{hw}}}{1+T_{\mathrm{rec}}^{\prime} s} \\
\frac{\Delta T_{h}(s)}{\Delta I(s)}=\frac{K_{\mathrm{rec}} K_{\mathrm{con}}}{1+T_{\mathrm{rec}}^{\prime} s} .
\end{gathered}
$$

Similarly, using (2), (3), (5), (7), and (8), the following small-signal equations can also be obtained:

$$
\begin{gathered}
\Delta \dot{p}_{\text {mean }}=\Delta(g A) \\
\Delta \tau_{m}=K_{\mathrm{mp}} \Delta p_{\text {mean }}+K_{\mathrm{mw}} \Delta \omega_{m} \\
T_{v} \Delta(g \dot{A})=-\Delta(g A)+K_{v} \Delta c \\
2 H^{\prime} \Delta \dot{\omega}_{m}=\Delta \tau_{m}-\Delta \tau_{e}-F^{\prime} \Delta \omega_{m}
\end{gathered}
$$

where the expressions for the parameters $K_{\mathrm{hp}}, K_{\mathrm{hw}}, K_{\mathrm{mp}}$, $K_{\mathrm{mw}}, T_{\mathrm{sel}}$, and $T_{\mathrm{rec}}^{\prime}$ are given in Appendix B.

Equations (9) and (13)-(16) constitute the linearized smallsignal model of the DS-DFIG power plant which can be used for controller design. The fourth-order model has two inputs $\Delta c$ and $\Delta \tau_{e}$, and two outputs $\Delta T_{h}$ and $\Delta \omega_{m}$, while $\Delta I$ is the disturbance. Classical speed control technique of DFIG is well-established and the technique can reject the torque disturbance $\Delta \tau_{m}$ introduced by the prime mover. However, the traditional mean pressure control technique for regulating the DS temperature is designed without considering speed variation $\Delta \omega_{m}$. As shall be shown later, $\Delta \omega_{m}$ does contribute toward $\Delta T_{h}$. Thus, in this investigation, the speed controller will be designed first and then the temperature controller can be designed by considering the speed as a disturbance, as shown in the following sections.

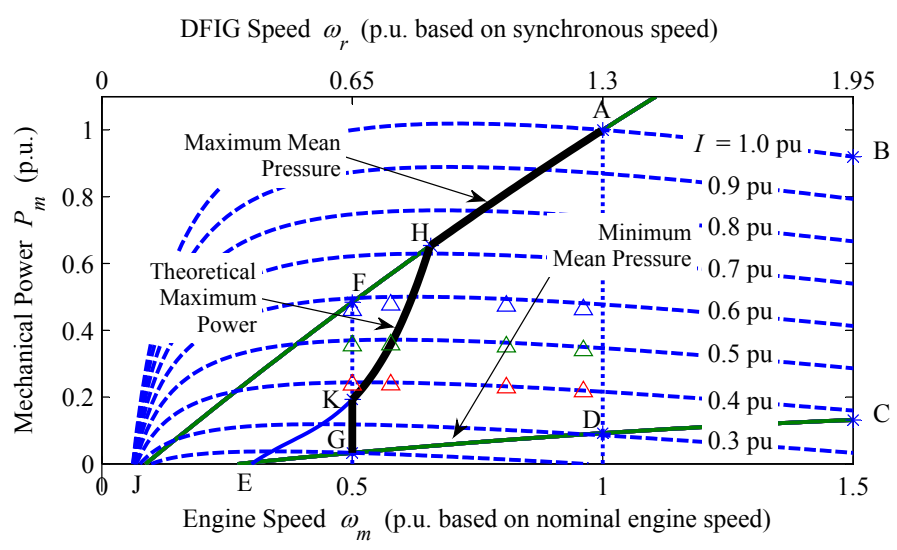

Fig. 3. Operating region and MPPT curve of DS-DFIG system.

\section{Speed Control of DFIG}

In this section, speed control of the DS-DFIG shall be considered for the purpose of maximizing its output power.

\section{A. Optimal Stirling Engine Speed for Maximum Output Power}

The expression for the steady-state mean pressure of the working gas can be obtained by substituting (1) into (6) and let $\dot{T}_{h}=0$, thus

$$
p_{\text {mean }}=\frac{K_{\mathrm{con}} I-a_{01} \omega_{m}-a_{00}-\left(1-T_{a}\right) / K_{\mathrm{rec}}}{a_{11} \omega_{m}+a_{10}} .
$$

In arriving at (17), one assumes the temperature is maintained at the maximum value, i.e., $T_{h}=1.0$ p.u. This is because the thermal efficiency of the engine increases with the temperature of the working gas. However, the temperature must not exceed a pre-set limit so as to prevent damage to the absorber tubes. Substituting (17) into (2), one can obtain a family of the steady-state mechanical power $P_{m}$ vs. engine speed $\omega_{m}$ curves at various solar irradiance levels $I$, as shown in Fig. 3.

The steady-state operations of the DS-DFIG system are somewhat constrained due to practical limits placed on the mean pressure $p_{\text {mean }}$ of the working gas and $I$. The constraints have to be reflected in Fig. 3. In Fig. 3, the $P_{m}-\omega_{m}$ curve under maximum $p_{\text {mean }}$ operation is shown by the line A-J whereas that at the minimum $p_{\text {mean }}$ is the curve C-E. A-B is part of the $P_{m}-\omega_{m}$ curve corresponding to the maximum $I$. Furthermore, as the operating speed range of a Stirling engine is typically much wider than that of a DFIG, feasible speed range of a DS-DFIG system is thus constrained by the speed range of the DFIG. Typically, DFIG operates within the range of 0.65 to 1.3 p.u. of the synchronous speed. If no spinning reserve or frequency support is required from the DS-DFIG, the nominal Stirling engine speed can be designed to match the maximum DFIG speed, i.e., 1.3 p.u. of the synchronous speed. Thus, the corresponding minimum engine speed is reduced to $\omega_{m, \min }=0.65 / 1.3=0.5$ p.u. of the nominal engine speed. Thus, the feasible steady-state operating engine speed is between 0.5 to 1 p.u. of the nominal engine speed. By incorporating this engine speed range constraint into the $P_{m}$ $-\omega_{m}$ plot, it is clear that the feasible operating state of the 


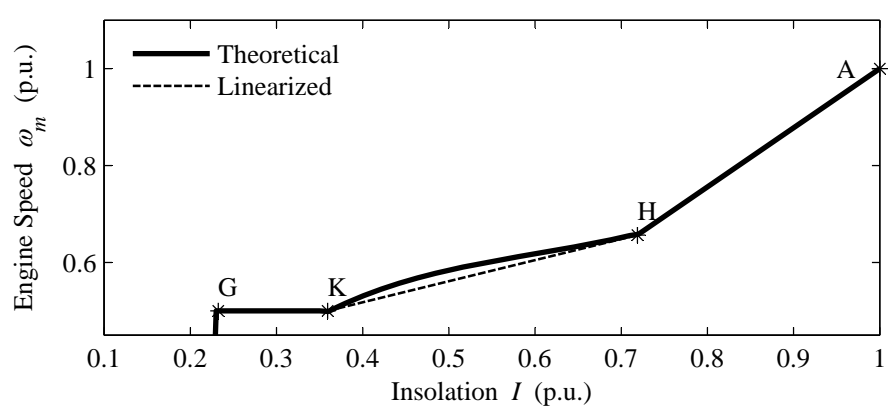

Fig. 4. Steady-state relationship between optimal engine speed and insolation.

DS-DFIG system is within the area A-D-G-F-A shown in Fig. 3.

Within the feasible operating area, maximum power harness can be realized by the control of the engine/generator speed. In Fig. 3, the maximum power point tracking (MPPT) locus is indicated by the line $\mathrm{A}-\mathrm{H}-\mathrm{K}-\mathrm{G}$ which is made up of three line sections: A-H governs the maximum power harness under relatively high $I$ but is restricted by the maximum $p_{\text {mean }}$ limit of the Stirling engine, $\mathrm{H}-\mathrm{K}$ is the theoretical MPPT locus at intermediate $I$ level, and when $I$ drops below the level corresponding to the point $\mathrm{K}$, the speed of the DFIG reaches its minimum limit.

For a given $I$, the corresponding speed on the MPPT curve is defined as the optimal speed $\omega_{m, \text { opt }}(I)$. Since A-H-K-G is a known function of $I, \omega_{m, \text { opt }}(I)$ can be calculated. So for realtime MPPT application, the engine speed can be regulated to track $\omega_{m, \text { opt }}(I)$ since $I$ can be measured on-line and is known.

Denote the MPPT locus by the function $P_{m, \mathrm{opt}}=$ $f_{\text {mppt }}\left(\omega_{m, \text { opt }}\right)$. Substituting (17) into (2), the steady-state relationship between $\omega_{m \text {,opt }}$ and $I$ can be shown to be

$$
\begin{gathered}
P_{m, \mathrm{opt}}=f_{\mathrm{mppt}}\left(\omega_{m, \mathrm{opt}}\right)=\left(b_{00}+b_{01} \omega_{m, \mathrm{opt}}+b_{02} \omega_{m, \mathrm{opt}}^{2}\right) \\
+\left(b_{10}+b_{11} \omega_{m, \mathrm{opt}}+b_{12} \omega_{m, \mathrm{opt}}^{2}\right) \frac{K_{\mathrm{con}} I-a_{01} \omega_{m, \mathrm{opt}}-a_{00}^{\prime}}{a_{11} \omega_{m, \mathrm{opt}}+a_{10}^{\prime}}
\end{gathered}
$$

The $\omega_{m, \text { opt }}-I$ curve corresponding to the MPPT curve AH-K-G is shown in Fig. 4. From (18) and Fig. 4, it can be seen that $\omega_{m, \text { opt }}$ is a complex and nonlinear function of $I$. For the purpose of analysis and control system design, this curve can be approximated by three linear sections: A-H corresponds to the maximum pressure section, $\mathrm{H}-\mathrm{K}$ is the theoretical MPPT section while $\mathrm{K}-\mathrm{G}$ is that of the minimum speed. For smallsignal analysis, revised MPPT curve can be expressed as

$$
\Delta \omega_{m}^{*}=\Delta \omega_{m, \mathrm{opt}}=k_{\mathrm{mppt}} \Delta I
$$

where $k_{\mathrm{mppt}}$ is the slope of the $\omega_{m, \mathrm{opt}}-I$ curve at the corresponding insolation level. Equation (19) indicates that one only needs to set the engine speed reference signal $\omega_{m}^{*}$ in the DS speed control system to $\omega_{m, \text { opt }}$ in order to achieve MPPT.

\section{B. Current and Speed Control of DFIG}

As explained in Section II.B, $\tau_{e}$ is assumed to be the same as its reference value $\tau_{e}^{*}$. Without any loss of generality, stator

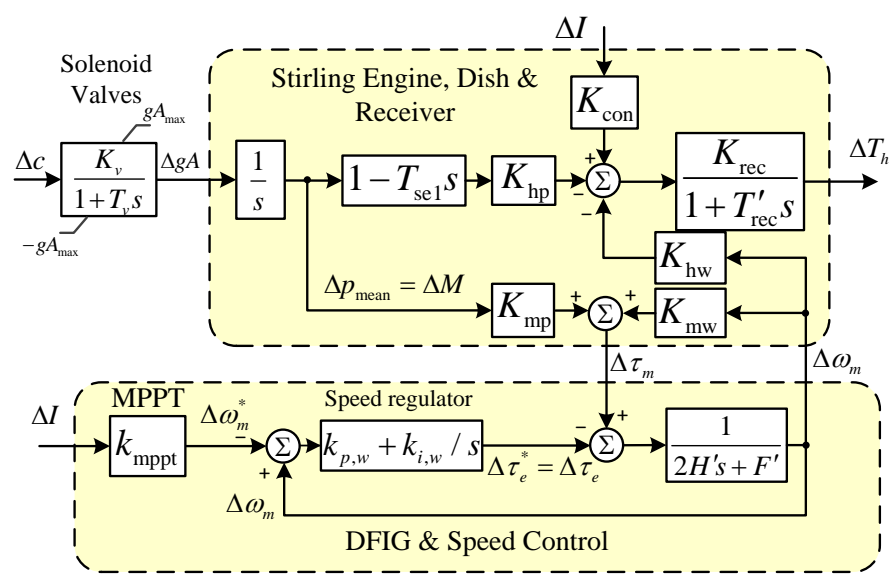

Fig. 5. Linearized DS-DFIG plant model for temperature control system design.

voltage oriented vector control (SVOC) is considered. The $d$ axis rotor current $i_{\mathrm{dr}}$ is proportional to the electromagnetic torque $\tau_{e}$. Torque reference $\tau_{e}^{*}$ is generated as the output of a speed regulator, and the corresponding $d$-axis current reference is

$$
i_{\mathrm{dr}}^{*}=\frac{L_{s}}{\psi_{s} L_{m}} \tau_{e}^{*}
$$

Various speed/power control strategies for DFIG have been discussed in [10] and they would be suitable for use in the DS-DFIG system. In Fig. 5, a closed-loop speed PI controller is used. The speed reference $\omega_{m}^{*}$ obtained from the $\omega_{m, \text { opt }}-I$ curve shown in Fig. 4 or (19) is compared with the measured engine/generator speed, and the error signal is sent to the speed regulator. $k_{p, w}$ and $k_{i, w}$ of the PI controller can be tuned using classical control techniques. The small-signal representation of the speed regulator is

$$
\begin{gathered}
\Delta \tau_{e}^{*}=k_{p, w}\left(\Delta \omega_{m}-\omega_{m}^{*}\right)+\Delta z \\
\Delta \dot{z}=k_{i, w}\left(\Delta \omega_{m}-\omega_{m}^{*}\right)
\end{gathered}
$$

where $z$ is the output of the integrator of the PI controller.

The control of $q$-axis rotor current $i_{\mathrm{qr}}$ depends on the strategy of reactive power/terminal voltage control. Design methods are available and interested readers may wish to refer to [10] and this aspect shall not be elaborated in this article.

\section{Temperature Control Under VARIABle-Speed OPERATION}

As explained in Section II-C, the speed control loop can reject the torque disturbance caused by temperature variation in the prime mover. Thus, the speed control system of DFIG can be designed first using well-established methods and the speed control loop becomes part of the whole plant for which the design of the temperature controller is to be dealt with next. The original plant model governed by (9), (13)-(16) is reduced to a single-input single-output (SISO) system with the disturbance $\Delta I$. The block diagram of the reduced DSDFIG plant model for temperature controller design is shown in Fig. 5. 


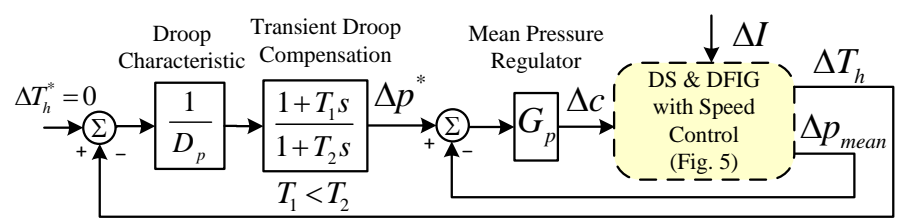

Fig. 6. Block diagram of classical control method with droop characteristic.

\section{A. Temperature Control With Droop Characteristics}

Temperature control based on droop characteristic for the conventional constant-speed DS system is introduced in [3] and is discussed in [2], [4]. The block diagram of the overall control system is shown in Fig. 6 which shows an inner loop where the mean pressure of the working gas is regulated by the proportional controller $G_{p}$. The outer loop includes the droop setting block. The droop characteristic introduces steady-state error of the temperature. The transient droop compensation is equivalent to a series lead-lag compensator which can improve damping for the nonminimum phase system [2]. The temperature of the receiver at periods of low $I$ would be lower than that at high $I$. So in order to increase the thermal efficiency of the DS system, the droop of the temperature controller needs to be reduced but this is at the expense of a deterioration in system damping. Steady-state error cannot be eliminated using the transient droop compensation technique [2]. Furthermore, the feedforward compensator based on speed input proposed in [2] is designed for constant-speed operation. This controller design approach is obviously unacceptable for the variable-speed operation and new temperature control schemes shall be examined next.

\section{B. Local State-Feedback With Integral Control}

In order to maximize the thermal efficiency of the DS-DFIG system, temperature control system with zero steady-state error performance is necessary. By incorporating (19), (21), and (22) into (9) and (13)-(16), the small-signal state-space model of an SISO system at operating point $\mathbf{x}=\mathbf{x}_{0}$ is obtained

$$
\left.\begin{array}{l}
\Delta \dot{\mathbf{x}}=\mathbf{A} \Delta \mathbf{x}+\mathbf{B} \Delta u+\Delta \mathbf{d} \\
\Delta y=\mathbf{C} \Delta \mathbf{x}
\end{array}\right\}
$$

where $\Delta \mathbf{x}=\left[\Delta(g A), \Delta p_{\text {mean }}, \Delta T_{h}, \Delta \omega_{m}, \Delta z\right]^{T}, \Delta u=\Delta c$, $\Delta \mathbf{d}=\left[0,0, K_{\mathrm{con}} K_{\mathrm{rec}} / T_{\mathrm{rec}}^{\prime}, k_{\mathrm{mppt}} k_{p, w} /\left(2 H^{\prime}\right),-k_{\mathrm{mppt}} k_{i, w}\right]^{T} \Delta I$, and $\Delta y=\Delta T_{h}$. Detailed expressions for the elements in matrices $\mathbf{A}, \mathbf{B}$, and $\mathbf{C}$ are given in Appendix $\mathbf{B}$ where it can be seen that the parametric values of elements of $\mathbf{A}$ are dependent of $K_{\mathrm{hp}}, K_{\mathrm{hw}}, K_{\mathrm{mp}}, K_{\mathrm{mw}}, T_{\mathrm{sel}}$, and $T_{\mathrm{rec}}^{\prime}$, which are in turn nonlinear functions of the state variables $p_{\text {mean }}$ and $\omega_{m}$, and thus $I$.

All the state variables in $\mathbf{x}$ are measurable: For example, thermocouple sensors, pressure gauges, and speed sensors are used to measure $T_{h}, p_{\text {mean }}$, and $\omega_{m}$, respectively, in the DS system described in [11], whereas $g A$ can be calculated as it is the derivative of $p_{\text {mean }}$, and $z$ can be generated as it is the output signal of integrator of the speed regulator. Thus, full state feedback control strategy can be realized to achieve temperature control. The design of the controller could be based on the placement of closed-loop poles in desirable locations in the complex plane. In addition, an integral control is introduced to eliminate the steady-state error in $T_{h}$ due to step change in $I$. The structure of the controller is thus

$$
\Delta u=-\mathbf{K}_{1} \Delta \mathbf{x}-K_{2} \int\left(\Delta y-\Delta y_{r}\right) d t
$$

where $y_{r}$ is the reference for $T_{h} . \mathbf{K}_{1}$ and $K_{2}$ are the control parameters of the state feedback and integral control loops respectively, and their values can be calculated using wellestablished pole-placement technique. Indeed, by following the analysis method shown in [12], it can be shown that if the following requirements are met, placement of the poles at any desirable locations in the complex plane is achievable and the DS heater temperature $T_{h}$ will reach its set value following a step disturbance of $I$. The requirements are as follows.

- The original system (23) is controllable;

- Augmented matrix $\mathbf{R}=\left[\begin{array}{ll}\mathbf{A} & \mathbf{0} \\ \mathbf{C} & \mathbf{0}\end{array}\right]$ is full rank.

Check for compliance of the two requirements can be achieved using existing computational tool such as MATLAB [13]. Whence from (23) and (24), the transfer function $g(s)$ between the output $y$ and the input reference $y_{r}$ can be derived

$$
g(s)=\Delta y(s) / \Delta y_{r}(s)=\mathbf{C}^{\prime}\left(s I-\mathbf{A}^{\prime}\right)^{-1} \mathbf{B}^{\prime}
$$

where $\quad \mathbf{A}^{\prime}=\left[\begin{array}{cc}\mathbf{A}-\mathbf{B K}_{\mathbf{1}} & -\mathbf{B} K_{2} \\ \mathbf{C} & 0\end{array}\right], \quad \mathbf{B}^{\prime}=$ $[0,0,0,0,0,-1]^{T}$, and $\mathbf{C}^{\prime}=[0,0,1,0,0,0]$. By selecting the closed-loop poles at $s=\lambda_{1}-\lambda_{6}, \mathbf{K}_{1}$ and $K_{2}$ can be obtained by solving

$$
\left|\mathbf{C}^{\prime}\left(s \mathbf{I}-\mathbf{A}^{\prime}\right)^{-1} \mathbf{B}^{\prime}\right|=\prod_{n=1}^{6}\left(s-\lambda_{n}\right) .
$$

There are various methods to obtain the solutions of (26). Interested readers may refer to [12] for the details.

\section{Fuzzy Supervisory Control Scheme}

In practice, $I$ tends to vary slowly compared to the thermodynamics of the Stirling engine and electromagnetic dynamics of the power system. Gain scheduling technique [14] can be adopted in this instance because it allows the most appropriate set of control parameters to be applied as the DS system operating states vary with the often random $I$ variations. Thus, a fuzzy supervisory control scheme is proposed which involves firstly the design of several local temperature controllers at selected steady-state operating points. The technique provides suitable control parameters for different operating conditions for corresponding insolation levels. The plant operating state can be characterized by $I(t)$, which is measured using insolation sensors such as pyranometers. Specially in this investigation, the insolation level is partitioned into three operating sections. For each section, a corresponding controller is designed using the full state feedback and integral control method described earlier. A Takagi-Sugeno fuzzy supervisor is used to calculate the average output of the controllers according to the measured $I$. Thus, with three triangular membership 
functions $\mu^{\text {(low })}, \mu^{\text {(medium) }}$, and $\mu^{\text {(high) }}$ to characterize $I$, the global output of the controllers can be expressed as

$$
u=\mu^{(\text {low })} u^{(\text {low })}+\mu^{(\text {medium })} u^{(\text {medium })}+\mu^{(\text {high })} u^{(\text {high })}
$$

where $u^{(\text {low })}, u^{\text {(medium) }}$, and $u^{(\text {high })}$ are the output of the controllers designed for $I=0.3,0.6$, and 0.9 p.u. respectively.

\section{ILlustrative EXAMPLE}

\section{A. Simulation Model of the Test System}

In this example, a 9-MW DS-DFIG system is assumed connected to a large grid through an equivalent impedance of $0.005+j 0.028$ p.u., on 100 MVA base. The DFIG is represented by a 5 th-order model [15]. The back-to-back voltage source converters are modeled without the consideration of harmonics and losses. The prime mover is represented by the improved average-value model presented in Section II-A. Parameters of the DS-DFIG, converters and the control systems are given in Appendix C. The overall control system of the RSC and the temperature control is shown in Fig. 7. Simulation was carried out in MATLAB/Simulink.

Before applying the control scheme proposed in Sections IV-B and IV-C, three sets of the system matrices $\mathbf{A}, \mathbf{B}$, and C corresponding to the three selected operating irradiance levels $I=0.3,0.6$, and 0.9 p.u were calculated based on the system parametric values given in Appendix C. Furthermore, using MATLAB, it was verifed that the system is controllable and the augmented matrix $\mathbf{R}$ is of full rank. Thus, the fuzzy supervisory control scheme can be realized, based on the fullstate feedback control with integral control. Complete pole placement is feasible. Using (25) and (26), the control parameters $\mathbf{K}_{1}$ and $K_{2}$ corresponding to the selected irradiance levels were calculated and they are also shown in Appendix C.

\section{B. Case 1: Transient Response Under Insolation Change}

This example is to compare the dynamic performances of the temperature controllers designed using different approaches. Fig. 8 shows the engine speed $\omega_{m}$ and temperature $T_{h}$ responses following a 0.05 -p.u. step increase of $I$ at the initial operating point $I_{0}=0.9$ p.u. The control parameters of the state-feedback controller is tuned at $I=0.6$ p.u. Hence, it is not surprising to note that the perturbations in $T_{h}$ are poorly damped. On the other hand, the fuzzy supervisory controller is seen to provide much improved dynamic performance, as it is able to adjust the control parameters according to the measured insolation level.

Fig. 9 shows the dynamic response of the DS-DFIG system when $I$ is assumed to increase from 0.25 p.u. to 1.0 p.u. at a rate of 0.1 p.u./s, i.e., $100 \mathrm{~W} /\left(\mathrm{m}^{2} \cdot \mathrm{s}\right)$. Normally, a rate of $30 \mathrm{~W} /\left(\mathrm{m}^{2} \cdot \mathrm{s}\right)$ is considered to be fast [16] and hence, this is an extremely strenuous test. It can be seen as $I$ increases from 0.3 to 1.0 p.u., the engine accelerates from 0.5 p.u. to 1.0 p.u. of nominal engine speed, i.e., from the $-35 \%$ to $+30 \%$ of the synchronous speed. With droop controller, the temperature is not at the maximum level until $I$ is at the maximum. Hence, the droop controller cannot ensure the DS-DFIG system is operating at the maximum efficiency level. This short-coming is alleviated using the state feedback
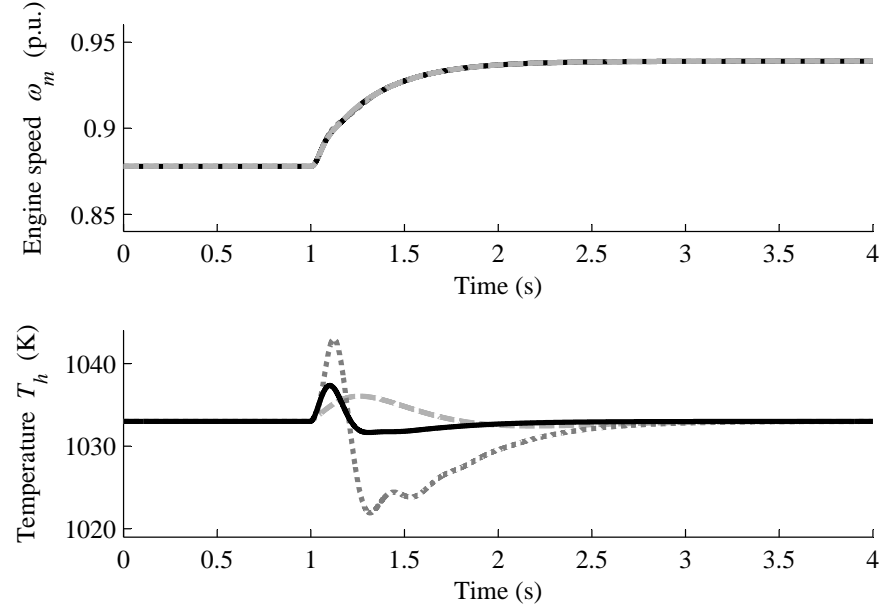

Fig. 8. DS-DFIG responses to 0.05 p.u. step increase in $I$ at $I_{0}=0.9$ p.u. Solid line: fuzzy supervisory control; dashed line: droop control; dotted line: full state feedback control tuned at $I=0.6$ p.u. (medium level).
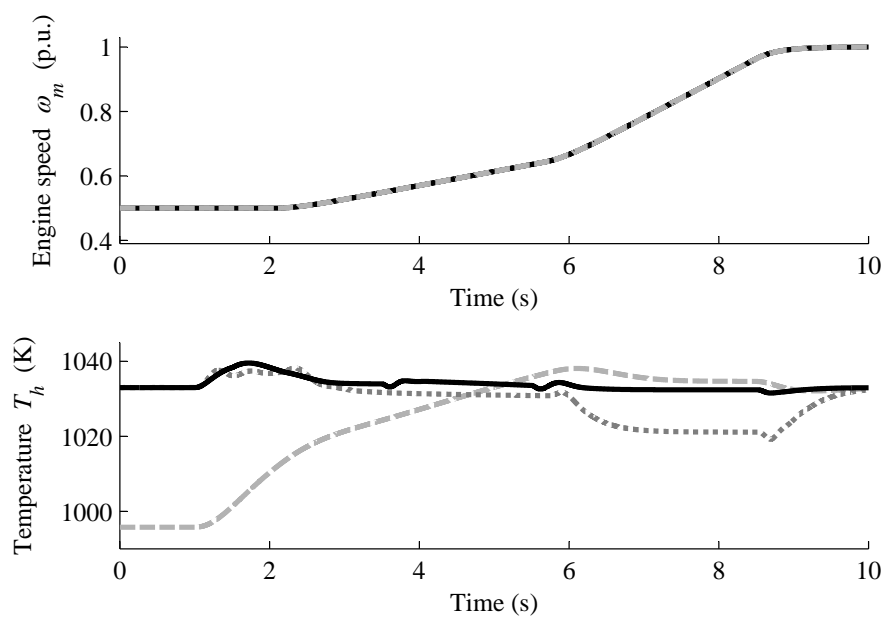

Fig. 9. DS-DFIG responses to a ramp increase in $I$ at 0.1 p.u./s from $I=0.25$ p.u. to 1.0 p.u. Solid line: fuzzy supervisory control; dashed line: droop control; dotted line: full state feedback control tuned at $I=0.6$ p.u. (medium level).

and fuzzy supervisory controllers as they allow the steadystate error in the temperature to be reduced. As the state feedback controller with a single integrator can only eliminate the steady-state errors following a step change disturbance, temperature rises during the ramp change of $I$. The maximum temperature rise depends on the maximum rate of change of $I$. From Fig. 9, although all the controllers have produced similar dynamic performances at low and medium $I$ levels, the fuzzy supervisory controller can maintain the temperature at the set value more effectively than the state feedback controller at higher $I$ levels.

\section{Case 2: Maximum Power Point Tracking}

This example is to demonstrate the maximum power tracking capability of the designed system. For such long-term studies, steady-state analysis was carried out. Generated powers from the proposed DS-DFIG MPPT scheme and traditional 


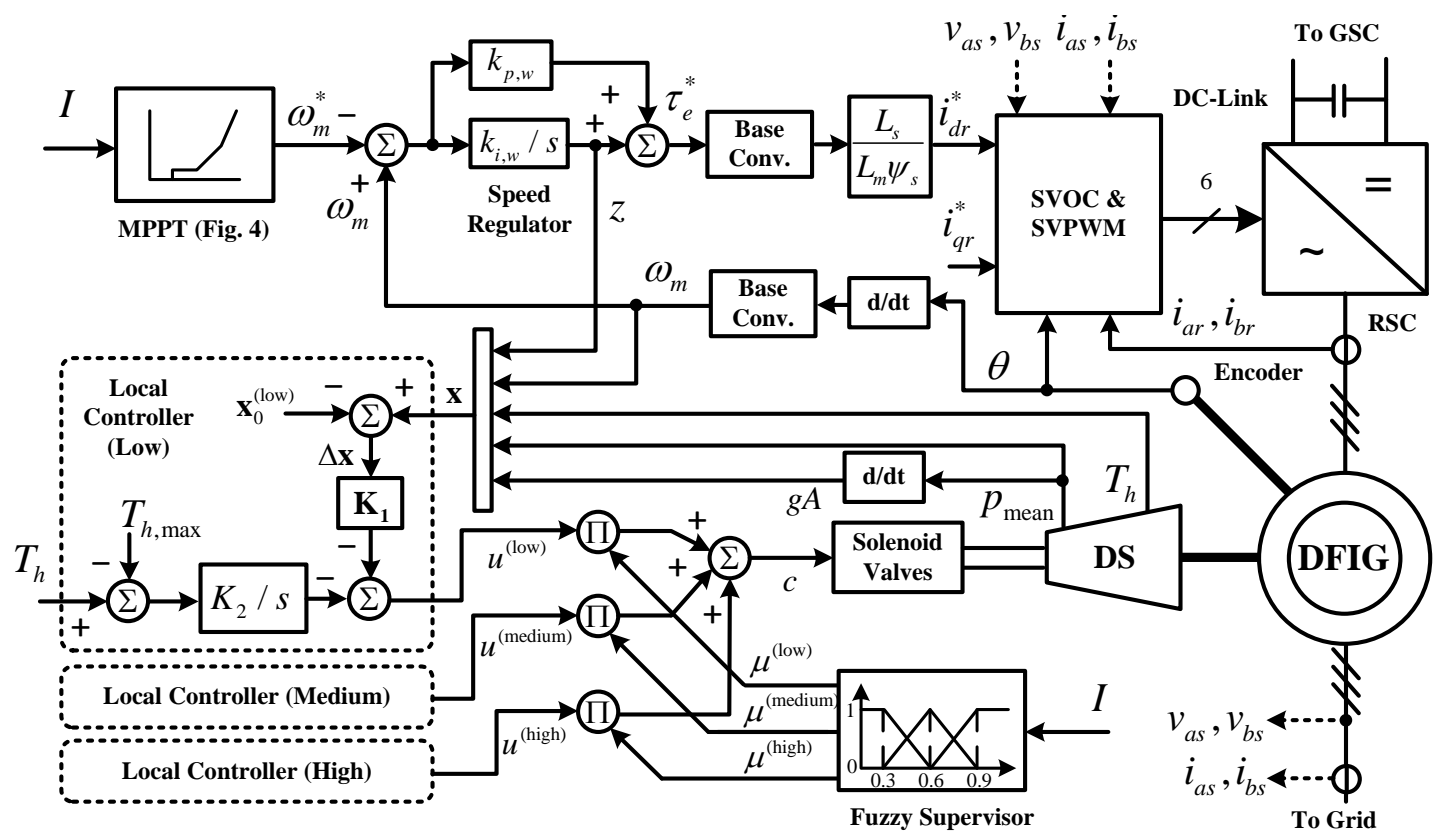

Fig. 7. Overall control scheme for the DS-DFIG system.

constant-speed DS system described in [2] are compared. In this example, the hourly measured insolation level of 2005 and that of every minute on Aug 5 and 6, 2005 were downloaded from [17]. The location where the measurements were taken is latitude $45.587^{\circ}$ and longitude $10.44^{\circ}$. Aug 52005 was a clear day whereas it was cloudy on the next. Figs. 10(a) and (b) show the generated power on the clear and cloudy days respectively. It can be seen that through the proposed MPPT scheme, the DS-DFIG system is able to extract more power and hence, more energy from the sun. In this case, the energy harness increases by $3.63 \%$ and $10.83 \%$ in the clear and cloudy days respectively. From the number of hours vs insolation level distribution profile shown in Fig. 10(c), the total amount of energy harness in 2005 can be calculated under the proposed DS-DFIG MPPT and constant-speed operations. In this example, the proposed MPPT scheme can increase energy harness by about $5.6 \%$.

\section{EXPERIMENTAL Results of MAXIMUM Power TRACKING OPERATION OF DS-DFIG}

A DS-DFIG emulator has been developed in the authors laboratory, with the view to study the maximum energy harness ability of the DS-DFIG system. The developed system is based on the use of a separately-excited dc motor to generate the equivalent mechanical torque from the DS under specific $I$ level and engine/motor speed. This approach is similar to that often used in the studies of variable-speed wind power generation systems [9].

The required mechanical torque $\tau_{m}^{*}$ under insolation $I$ and engine speed $\omega_{m}$ can be calculated using (2), (5), and (17), i.e.,

$$
\tau_{m}^{*}=\sum_{i=0}^{1} \sum_{j=0}^{2} b_{i j}\left(\frac{K_{\mathrm{con}} I-a_{01} \omega_{m}-a_{00}-\frac{1-T_{a}}{K_{\mathrm{rec}}}}{a_{11} \omega_{m}+a_{10}}\right)^{i} \omega_{m}^{j-1} .
$$

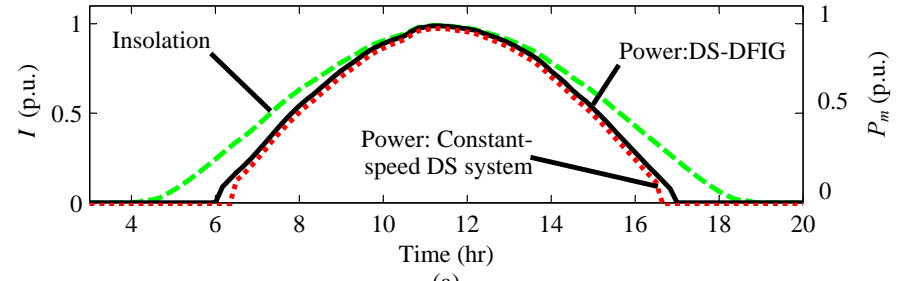

(a)

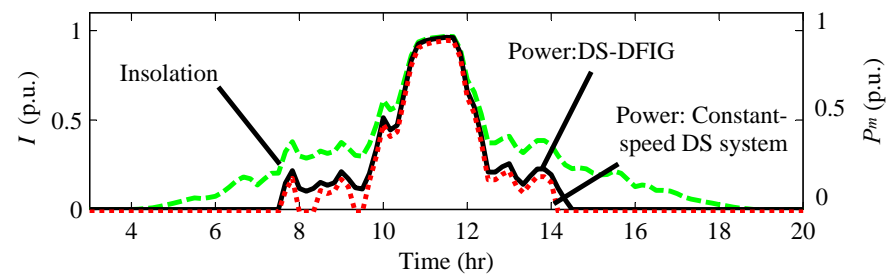

(b)

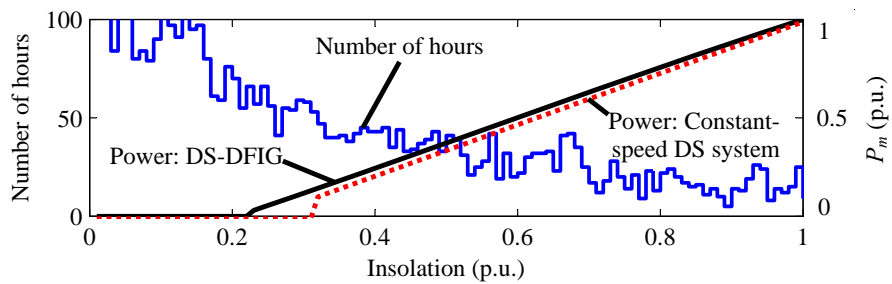

(c)

Fig. 10. Comparison of generated power between variable- and constantspeed DS system.

In the experimental setup, the dc armature voltage $V_{a}$ is manually varied, instead of using a dc drive, so as to reduce the hardware requirements. The steady-state relationship between the mechanical torque $\tau_{m}$, dc motor speed $\omega_{m}$ and dc armature voltage $V_{a}$ can be readily shown to be

$$
V_{a}=\frac{K_{e} \omega_{m} \omega_{m, N}+\sqrt{\tau_{m} \omega_{m} P_{m, N} R_{a}}}{2}
$$




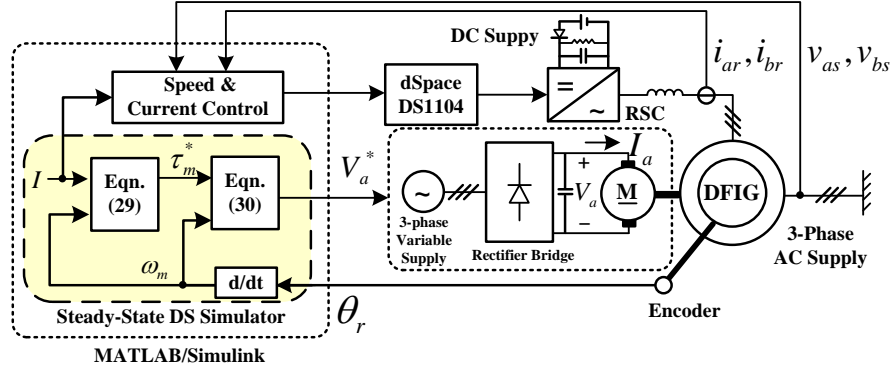

Fig. 11. Schematic of experimental setup for DS-DFIG system.

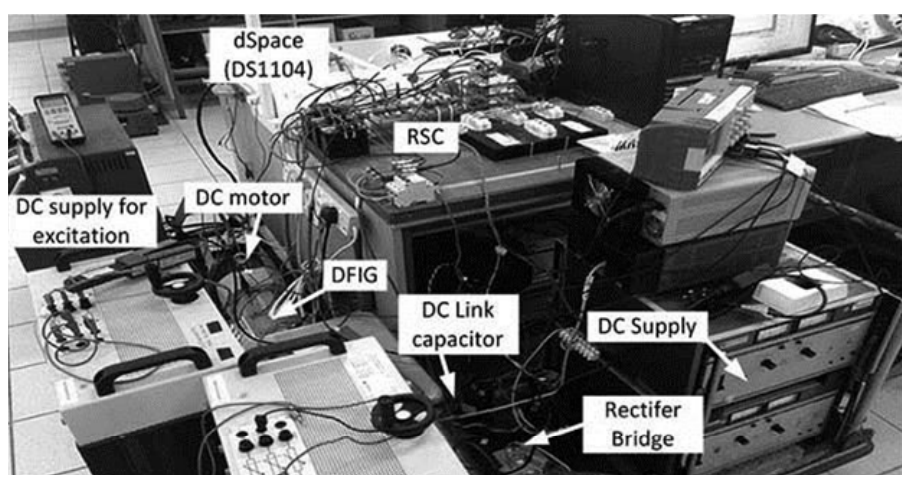

Fig. 12. Experimental setup of the DS-DFIG system with DS simulator.

where $K_{e}$ is the torque constant and $R_{a}$ is the armature resistance of the dc machine. The mechanical power is assumed to be the same as the input dc power of dc motor. Thus, using (28) and (29), the required dc armature voltage $V_{a}$ can be calculated for the steady-state DS simulator.

The schematic of the constructed DS-DFIG system is shown in Fig. 11. The speed and current control system of DFIG, (28) and (29) were implemented under dSpace (DS1104) platform, with MATLAB/Simulink in real-time interface. A $1.0-\mathrm{kW}$ separately-excited dc motor is connected to a $1.0-\mathrm{kW}$ DFIG with a RSC. Fig. 12 is a photograph of the experimental setup. Samples of the steady-state experimental results are shown in Table I. The measured results are normalized using the based values of DFIG and compared with the theoretical results, as shown in Fig. 3. It can be seen that the measured results (identified by the symbol $\Delta$ ) are in close agreement with those obtained from the theoretical analysis.

TABLE I

Results ObTaINED Under STEADY-STATE OPERATION OF DS-DFIG

\begin{tabular}{ccccc}
\hline \hline$I$ (p.u.) & $\omega_{m}$ (p.u.) & $\tau_{m}$ (p.u.) & $V_{a}(\mathrm{~V})$ & $P(\mathrm{~W})$ \\
\hline 0.6 & 0.65 & 0.9677 & 158.5 & 743.8 \\
0.6 & 0.75 & 0.8663 & 176.6 & 749.7 \\
0.6 & 1.05 & 0.6112 & 233.6 & 740.7 \\
0.6 & 1.25 & 0.5003 & 273.0 & 721.6 \\
0.5 & 0.65 & 0.7399 & 158.5 & 554.9 \\
0.5 & 0.75 & 0.6443 & 176.6 & 557.6 \\
0.5 & 1.05 & 0.4493 & 233.6 & 544.3 \\
0.5 & 1.25 & 0.3643 & 273.0 & 525.4 \\
0.4 & 0.65 & 0.4881 & 147.1 & 366.1 \\
0.4 & 0.75 & 0.4224 & 166.6 & 365.5 \\
0.4 & 1.05 & 0.2875 & 226.3 & 348.3 \\
0.4 & 1.25 & 0.2283 & 273.0 & 329.3 \\
\hline \hline
\end{tabular}

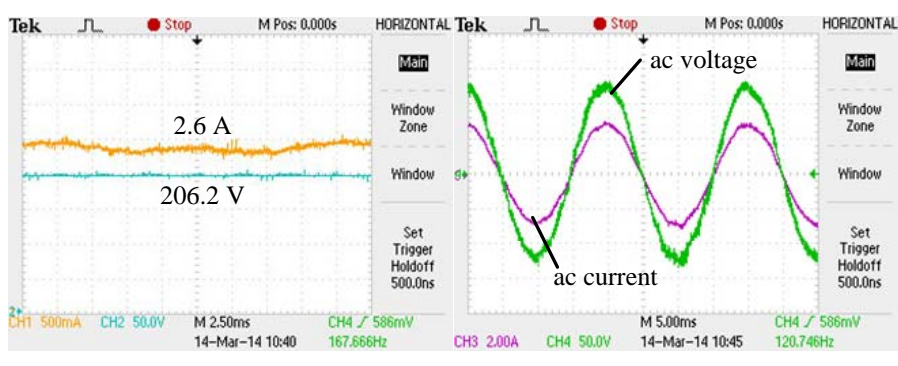

(a)

(b)

Fig. 13. Experimental results obtained at maximum power operation when $I=0.626$ p.u. (a) dc armature voltage and current (b) ac stator voltage and current.

Samples of the steady-state waveforms of the armature voltage and current are shown in Fig. 13. It shows that at $I=0.626$ p.u., the DFIG speed is controlled at the optimal speed $\omega_{m, \text { opt }}=0.75$ p.u. By regulating the armature voltage at $206.2 \mathrm{~V}$, as determined by (28) and (29), the measured armature current is about $2.6 \mathrm{~A}$. The resulting power of 0.536 p.u. (on $1 \mathrm{~kW}$ base) compares favourably with the value calculated using (18), which is 0.5338 p.u.

\section{CONCLUSIONS}

An improved model of the Stirling engine has been derived for the study of variable-speed operation of the DS system. The proposed DS-DFIG system is to realize maximum energy harness. A supervisory fuzzy control scheme has been identified as a suitable means because it can effectively mitigate the impacts of the engine speed variations on the DS receiver temperature. Simulation and experimental results have demonstrated the effectiveness of the proposed approach in maximizing the solar energy harness while ensuring satisfactory control of the DS-DFIG receiver temperature.

\section{APPENDIX A}

In [2], an average-value model of the Stirling engine was derived using ideal adiabatic analysis. However, in arriving at this model, the engine speed was assumed constant. Moreover, the rate of change of the temperature was assumed to be much slower than that of the pressure, i.e., $\mathrm{D} T / T \ll \mathrm{D} p / p$. These assumptions may not be valid for the variable-speed operation. Thus, in this appendix, an improved average-value model of the engine for variable-speed operation will be developed. Note the meaning of the symbols used herewith were explained in [2] and would not be repeated here due to space constraint.

The mass flow equations of the ideal adiabatic model considering temperature variation are

$$
\begin{gathered}
\mathrm{D} m_{h}=m_{h}\left(\mathrm{D} p / p-\mathrm{D} T_{h} / T_{h}\right) \\
g A_{\mathrm{rh}}=g A_{\mathrm{he}}+\mathrm{D} m_{h}=\mathrm{D} m_{e}+\mathrm{D} m_{h} .
\end{gathered}
$$

Substituting (30) into (31), and using the ideal gas equation, one can obtain

$$
g A_{\mathrm{rh}}=\mathrm{D} m_{e}+\left(V_{h} / R T_{h}\right) \mathrm{D} p-\left(V_{h} / R T_{h}^{2}\right) p \mathrm{D} T_{h} .
$$

Unlike [2] where the third term of (32) is ignored by assuming $\mathrm{D} T_{h} / T_{h} \ll \mathrm{D} p / p$, this term is retained and the total 
heat absorbed by four cylinders of the double-acting kinematic Stirling engine is

$$
\begin{gathered}
\mathrm{D} Q_{h, \text { total }}=\sum_{i=1}^{4} \mathrm{D} Q_{h, i}=-V_{h} \overbrace{\sum_{i=1}^{4} \mathrm{D} p_{i}}^{1}-c_{p} T_{h} \overbrace{\sum_{i=1}^{4} \mathrm{D} m_{e, i}}^{(2)} \\
+\frac{c_{p}}{R} \overbrace{\sum_{i=1}^{4}\left(p_{i} \mathrm{D} v_{e, i}+v_{e, i} \frac{\mathrm{D} p_{i}}{\gamma}\right)}^{\text {(3) }}+\overbrace{\frac{c_{p} V_{h}}{R} \overbrace{\sum_{i=1}^{4}\left(\frac{\left.\mathrm{D} T_{h, i} p_{i}\right)}{\left.T_{h, i}\right)}\right.}^{(4)}}^{\text {(33) }}
\end{gathered}
$$

where $i=1-4$ indicates the cylinder number.

First, (1) and (3) in (33) have already been derived in [2], i.e.,

$$
\begin{gathered}
\sum_{i=1}^{4} \mathrm{D} p_{i}=4 \mathrm{D} p_{\text {mean }} \\
\sum_{i=1}^{4}\left(p_{i} \mathrm{D} v_{e, i}+v_{e, i} \frac{\mathrm{D} p_{i}}{\gamma}\right)=\frac{4 R}{c_{p}} V_{1} p_{\text {mean }}+\frac{4 c_{v}}{c_{p}} V_{3} \mathrm{D} p_{\text {mean }}
\end{gathered}
$$

Next, (4) in (33) can be readily evaluated as

$$
\sum_{i=1}^{4} \frac{\mathrm{D} T_{h, i}}{T_{h, i}} p_{i} \approx \frac{p_{\text {mean }}}{T_{h, \text { max }}} \mathrm{D} T_{h}
$$

Finally, since the assumption $\mathrm{D} T_{h} / T_{h} \ll \mathrm{D} p / p$ is not valid, (2) in (33) can be evaluated in the following way.

$$
\mathrm{D} m_{e, i}=\mathrm{D}\left(\frac{p_{i} v_{e, i}}{R T_{e, i}}\right) \approx \frac{p_{i} v_{e, i}}{R T_{h}}+\frac{v_{e, i} \mathrm{D} p_{i}}{R T_{h}}-\frac{p_{i} v_{e, i} \mathrm{D} T_{h}}{R T_{h}^{2}}(37)
$$

Thus, the summation of (37) is

$$
\sum_{i=1}^{4} \mathrm{D} m_{e, i}=\frac{1}{R T_{h}}\left(\sum_{i=1}^{4} p_{i} \mathrm{D} v_{e, i}+\sum_{i=1}^{4} v_{e, i} \mathrm{D} p_{i}-\frac{\mathrm{D} T_{h}}{T_{h}} \sum_{i=1}^{4} p_{i} v_{e, i}\right) .
$$

From [2],

$$
\begin{gathered}
\sum_{i=1}^{4} p_{i} \mathrm{D} v_{e, i}=4 V_{1} p_{\text {mean }} \\
\sum_{i=1}^{4} v_{e, i} \mathrm{D} p_{i}=4 V_{2} p_{\text {mean }}+4 V_{3} \mathrm{D} p_{\text {mean }} \\
\sum_{i=1}^{4} p_{i} v_{e, i}=4 V_{3} p_{\text {mean }}
\end{gathered}
$$

where $V_{1}=-0.25 V_{\mathrm{sw}} b \cos \theta, V_{2}=0.25 V_{\mathrm{sw}} b \cos \theta, V_{3}=V_{d}+$ $0.25 V_{\mathrm{sw}} b \sin \theta, V_{d}=V_{\mathrm{cl}}+0.5 V_{\mathrm{sw}}$. Thus, substituting (39)-(41) into (38), (2) becomes

$$
\sum_{i=1}^{4} \mathrm{D} m_{e, i}=\frac{4 V_{3}}{R T_{h}}\left(\mathrm{D} p_{\text {mean }}-\frac{p_{\text {mean }}}{T_{h}} \mathrm{D} T_{h}\right)
$$

Then substituting (34)-(36) and (42) into (33), and assuming $T_{h}=T_{h, \text { max }}$, one obtains

$$
\mathrm{D} Q_{h, \text { total }}=4 V_{1} p_{\text {mean }}-4\left(V_{h}+V_{3}\right)\left(\mathrm{D} p_{\text {mean }}+\frac{c_{p} p_{\text {mean }}}{R T_{h, \text { max }}} \mathrm{D} T_{h}\right)
$$

The mean pressure of the working gas is proportional to the total mass of the working gas, i.e.,

$$
p_{\text {mean }}=K_{p} M
$$

where the coefficient $K_{p}$ can be seen as constant when the temperature is near the normal operation value.

The base values used to derive a normalized model are: $p_{\text {base }}=p_{\text {max }}$, the maximum mean pressure (expressed in Pa); $m_{\text {base }}=M_{\max }$, the total mass of the working gas when the pressure is maximum $(\mathrm{kg}) ; T_{\text {base }}=T_{h, \max }$, the maximum temperature of receiver $(\mathbf{K}) ; \omega_{\text {base }}=\omega_{m, N}$, the nominal engine speed $(\mathrm{rad} / \mathrm{s}) ; P_{\text {base }}=P_{m, N}$, the generated nominal mechanical power $(\mathrm{W})$. With the base values, the per-unit mean pressure would be equal to the per-unit total mass of the working gas, i.e.,

$$
\bar{p}_{\text {mean }}=\bar{M} .
$$

The overbar "-" indicates the normalized or per-unit quantities.

Considering (45) and using the base values to normalize (43), and converting it into time-domain expression, one obtains

$$
\dot{\bar{Q}}_{h, \text { adi }}=\bar{K}_{h} \bar{p}_{\text {mean }} \bar{\omega}_{m}+A \overline{g A}+C \bar{p}_{\text {mean }} \dot{\bar{T}}_{h}
$$

where $\bar{K}_{h}=\frac{4 p_{\max } \omega_{m, N}}{P_{m, N}} V_{1}, A=-\frac{4 p_{\max }}{P_{m, N}}\left(V_{h}+V_{3}\right), C=$ $\frac{4 p_{\max }}{P_{m, N}} \frac{c_{p}}{R}\left(V_{h}+V_{3}\right)=\frac{\gamma}{1-\gamma} A$, and $\overline{g A}=\dot{\bar{M}}=\dot{\bar{p}}_{\text {mean }}$.

The expression of mechanical power is the same as that in [2], i.e.,

$$
\bar{P}_{m, \text { adi }}=\bar{\tau}_{m} \bar{\omega}_{m}=\bar{K}_{m} \bar{p}_{\text {mean }} \bar{\omega}_{m}
$$

where $\bar{K}_{m}=\left(p_{\max } \omega_{m, N} / P_{m, N}\right) \sqrt{2} b \sin (\theta-0.75 \pi) V_{\text {sw }}$.

In the main text, the overbar "-" to indicate the normalized quantities will be omitted. Hence, (1)-(3) are derived.

\section{APPENDIX B}

$K_{\mathrm{hp}}=a_{10}+a_{11} \omega_{m 0}, K_{\mathrm{hw}}=a_{01}+a_{11} p_{0}, T_{\mathrm{sel}}=$ $-\eta_{h 0} A /\left(a_{10}+a_{11} \omega_{m 0}\right), T_{\mathrm{rec}}^{\prime}=T_{\mathrm{rec}}+\eta_{h 0} K_{\mathrm{rec}} C p_{0}, K_{\mathrm{mp}}=$ $b_{10} / \omega_{m 0}+b_{11}+b_{12} \omega_{m 0}, K_{\mathrm{mw}}=-b_{00} / \omega_{m 0}^{2}-b_{10} p_{0} / \omega_{m 0}^{2}+$ $b_{02}+b_{12} p_{0}$.

Elements of matrices $\mathbf{A}, \mathbf{B}$, and $\mathbf{C}$ are $A_{11}=$ $-1 / T_{v}, A_{21}=K_{\mathrm{rec}} K_{\mathrm{hp}} T_{\mathrm{sel}}, A_{31}=-K_{\mathrm{rec}} K_{\mathrm{hp}} / T_{\mathrm{rec}}^{\prime}, A_{32}=$ $-K_{\mathrm{rec}} K_{\mathrm{hp}} / T_{\mathrm{rec}}^{\prime}, A_{34}=-K_{\mathrm{rec}} K_{\mathrm{hw}} / T_{\mathrm{rec}}^{\prime}, A_{42}=$ $K_{\mathrm{mp}} /\left(2 H^{\prime}\right), A_{44}=\left(K_{\mathrm{mw}}-F^{\prime}-k_{p, w}\right) /\left(2 H^{\prime}\right), A_{45}=$ $-1 /\left(2 H^{\prime}\right), A_{54}=k_{i, w} \cdot B_{11}=K_{v} / T_{v}, C_{13}=1$, and remaining $A_{i j}=B_{i j}=C_{i j}=0$.

\section{APPENDIX C}

Parameters of the Prime Movers: $P_{m, N}=9 \mathrm{MW}, \omega_{m, N}=$ $190.63 \mathrm{rad} / \mathrm{s}, a_{00}=0.045, a_{10}=0.068, a_{01}=0.20, a_{11}=$ $2.14, b_{00}=-0.038, b_{10}=-0.072, b_{01}=0.055, b_{11}=1.21$, $b_{02}=-0.026, b_{12}=-0.13^{1}, A=-0.2735, C=0.8752$, $K_{\text {con }}=2.865^{1}, T_{\text {rec }}=13.436 \mathrm{~s}, K_{\text {rec }}=1.756^{1}, T_{a}=0.288$, $K_{v}=1.0$, and $T_{v}=0.02 \mathrm{~s}$.

Parameters of the DFIG and Converters: $S_{N}=10 \mathrm{MVA}$, $V_{N}=575 \mathrm{~V}, f_{N}=60 \mathrm{~Hz}, R_{s}=0.023$ p.u., $L_{s}=3.08$ p.u., $R_{r}=0.016$ p.u., $L_{r}=3.06$ p.u., $L_{m}=2.9$ p.u., $p_{n}=3$, $H=0.685 \mathrm{~s}$, and $F=0.01$.

Controller Parameters: $G_{p}=25, D_{p}=0.043, \mathbf{K}_{1}^{(\text {low })}=$ $[1.9,1046,-22485,-1384,-20.2], \quad K_{2}^{\text {(low) }}=-46057$,

\footnotetext{
${ }^{1}$ The numbers are corrected from the orginal publication.
} 
$\mathbf{K}_{1}^{\text {(medium) }}=[2,751,-20508,-1144,-19.8], K_{2}^{\text {(medium) }}=$ $-56509, \mathbf{K}_{1}^{(\mathrm{high})}=[2.3,708,-18888,-244,-5.8], K_{2}^{(\mathrm{high})}=$ $-59677, k_{p, w}=128$, and $k_{i, w}=4200$,

\section{REFERENCES}

[1] T. Mancini, P. Heller, B. Butler, B. Osborn, W. Schiel, V. Goldberg, R. Buck, R. Diver, C. Andraka, and J. Moreno, "Dish-Stirling systems: An overview of development and status," ASME J. Solar Energy Eng., vol. 125 , no. 2, pp. 135-151, May 2003.

[2] Y. Li, S. S. Choi, and C. Yang, "Dish-Stirling solar power plants: Modeling, analysis and control of receiver temperature," IEEE Trans. Sustain. Energy, vol. 5, no. 2, pp. 398-407, Apr. 2014.

[3] S. Almstrom, C. Bratt, and H. Neiving, "Control systems for United Stirling 4-95 engine in solar application," in Proc. Intersoc. Energy Convers. Eng. Conf., Atlanta, GA, USA, Aug. 1981, pp. 1888-1893.

[4] D. F. Howard, J. Liang, and R. G. Harley, "Control of receiver temperature and shaft speed in dish-Stirling solar power plants to meet grid integration requirements," in Proc. IEEE Energy Convers. Congr. Expo., Atlanta, GA, USA, Sep. 2010, pp. 398-405.

[5] F. J. G. Granados, M. A. S. Perez, and V. Ruiz-Hernandez, "Thermal model of the EuroDish solar Stirling engine," ASME J. Solar Energy Eng., vol. 130, no. 1, Feb. 2008.

[6] D. Howard and R. G. Harley, "Modeling of dish-Stirling solar thermal power generation," in Proc. IEEE Power Energy Soc. Gen. Meeting, Minneapolis, MN, USA, Jul. 2010, pp. 1-7.

[7] F. Nepveu, A. Ferriere, and F. Bataille, "Thermal model of a dish/Stirling systems," Sol. Energy, vol. 83, no. 1, pp. 81-89, Jan. 2009.

[8] D. Santos-Martin, J. Alonso-Martinez, J. Eloy-Garcia, and S. Arnalte, "Solar dish-Stirling system optimisation with a doubly fed induction generator," IET Renew. Power Gen., vol. 6, no. 4, pp. 276-288, Jul. 2012.

[9] R. Pena, J. C. Clare, and G. M. Asher, "Doubly fed induction generator using back-to-back PWM converters and its application to variable-speed wind-energy generation," Proc. IEE Elect. Power Appl., vol. 143, no. 3, pp. 231-241, May 1996.

[10] B. Wu, Y. Lang, N. Zargari, and S. Kouro, Power Conversion and Control of Wind Energy Systems. Hoboken, NJ, USA: Wiley, 2011.

[11] J. J. Droher and S. E. Squier, "Performance of the vanguard solar dishstirling engine module," Elect. Power Res. Inst., Palo Alto, CA, USA, Tech. Rep. EPRI AP-4608, Jul. 1986.

[12] R. Dorf and R. Bishop, Modern Control Systems. Englewood Cliffs, NJ, USA: Prentice Hall, 2008.

[13] MATLAB Control System Toolbox Reference, MathWorks, 2014.

[14] R. Palm, D. Driankov, and H. Hellendoorn, Model Based Fuzzy Control: Fuzzy Gain Schedulers and Sliding Mode Fuzzy Controllers. Bristol, U.K.: Springer, 1997.

[15] P. Kundur, N. Balu, and M. Lauby, Power System Stability and Control. New York, NY, USA: McGraw-Hill, 1994.
[16] S. B. Kjaer, "Evaluation of the "hill climbing" and the "incremental conductance" maximum power point trackers for photovoltaic power systems," IEEE Trans. Energy Convers., vol. 27, no. 4, pp. 922-929, Dec. 2012.

[17] "Time series of solar radiation data," 2005. [Online]. Available: http://www.soda-is.com/eng/services/services_radiation_free_eng.php

Yang $\mathbf{L i}$ (S'11) received the B.E. degree in electrical engineering in 2007 from Wuhan University, Wuhan, China, and the M.Sc. degree in power engineering from Nanyang Technological University, Singapore, in 2008, where he is currently working toward the Ph.D. degree.

His research interests include renewable energy, solar thermal power generation, and energy storage systems.

San Shing Choi (M'03-SM'07) received the B.E. and Ph.D. degrees from the University of Canterbury, Christchurch, New Zealand, in 1973 and 1976, respectively.

He was previously with the New Zealand Electricity Department, Wellington, New Zealand; the National University of Singapore, Singapore; and the State Energy Commission of Western Australia, Perth, Australia. He is now a Professor in the School of Electrical and Electronic Engineering, Nanyang Technological University, Singapore. His research interests include power system control, renewable, and energy storage systems.

Chun Yang received the B.Sc. degree from the Department of Thermal Engineering at Tsinghua University, Beijing, China, in 1985; M. Eng. degree in thermophysics from the University of Science and Technology of China, Hefei, China, in 1988; and the Ph.D. degree in mechanical engineering from University of Alberta, Edmonton, Canada, in 1999.

In 1999, he joined Nanyang Technological University, where he is currently an Associate Professor in the School of Mechanical and Aerospace Engineering. His current research interests include heat and mass transfer, electrokinetic transport phenomena, microfluidics, and microscale flow.

Feng Wei (S'10) received the B.E. degree in electrical engineering from Wuhan University, Wuhan, China, in 2008, and the M.Sc. degree, from Nanyang Technological University, Singapore, in 2009, where he is currently pursuing the Ph.D. degree.

His current research interests include wind power generation and electronic converters. 